\title{
Stress increases impulsive punishment avoidance in unmedicated mood disorders
}

Kristoffer C. Aberg, Rony Paz

Department of Brain Sciences, Weizmann Institute of Science, Israel

Correspondence should be addressed to: K.C.A (kc.aberg@gmail.com)

\section{Introduction}

In an intriguing study designed to test whether and how stress affects learning and decision making in anxious individuals, Aylward et al. (2019) ${ }^{1}$ used a restless four-armed bandit task, where threat of electric shocks was used to induce stress. They hypothesized that anxious individuals would learn faster from punishments under stress, and as a result, exhibit choices that are more affected by those punishments. Unfortunately, these hypotheses were not confirmed.

Here, we re-analyzed the original dataset using behavioral models that incorporate different mechanisms that could account for punishment-related behavioral biases. We found evidence that support the original hypotheses, namely increased punishment-avoidance for anxious individuals during stress. The avoidance-behavior was driven by punishments that were presented following a sequence of neutral outcomes, and in turn increased the tendency to elicit an immediate avoidance response (rather than larger learning rates). This impulsive avoidance bias was particularly pronounced in anxious individuals during stress.

Understanding the interaction between daily-life environment factors, such as stress, and the inter-individual expression of affective traits may inform the development of dysfunctional behavioral patterns and mental illness ${ }^{2-4}$. This research theme is particularly timely given reports of global increases in stress and mental health decline ${ }^{5-7}$. Importantly, by re-analyzing valuable data that was already collected, published, and made freely available, our study rectifies the original hypothesis, and thereby encourages future research on this highly relevant topic.

\section{Results}

Participants performed a four-armed restless bandit task with the goal of collecting happy smiley faces (rewards) and avoiding sad smiley faces (punishments) presented as feedback ${ }^{1}$. In each trial, participants selected one of the four bandits and probabilistic feedbacks were delivered at the selected bandit's spatial location (Fig.1A). In total, four feedbacks were provided (Fig.1B-E): reward and punishment together $(:):$ ), punishment-only $(:)$, reward-only $(\odot)$, and finally neither reward nor punishment (i.e. no faces were presented). Given the restless nature of the bandits, reward- and punishment probabilities varied dynamically across trials. To induce stress, participants were explicitly instructed that, unrelated to task-performance, electric shocks may be given at any time in the 'Threat' condition, but not in the 'Safe' condition. Two groups of 
participants were tested, one group of healthy controls $(n=88)$ and one group of participants with unmedicated mood and anxiety symptoms $(n=44)$.

\section{Original analysis}

To assess behavioral differences between Groups (Control, Anxious) and Condition (Threat, Safe), the original study compared how frequently participants re-selected a bandit after receiving reward-only $(:)$ ) or punishment-only $(*)$ feedbacks. The authors reported more repeated selections following reward-only, as compared to punishment-only feedback, but no main effects or interactions involving Group or Condition. As such, there was no evidence for an interaction between anxiety and stress on behavior.

\section{Separating rewards from punishments}

We first analyzed the proportion of repeated selections separately for each of the four feedback types using mixed-effect ANOVAs with Group and Condition as factors (Fig.1B-E), and applied a Bonferroni-corrected threshold for statistical significance $(\alpha=0.125)$. We found that the Group $x$ Condition interaction for punishment-only feedback was significant [Fig.1E; $F(1,130)=11.577$, $\left.\mathrm{p}<0.001, \eta_{p}^{2}=0.082\right]$. This interaction was caused by the Anxious group showing a higher proportion of rejections of bandits whose previous selections elicited a punishment-only feedback in the Threat versus the Safe condition [Fig.1G; Threat minus Safe for Anxious versus Controls: $t(130)=3.403, p<0.001,95 \% \mathrm{Cl}=0.0240 .092$, two-sample two-tailed $t$-test; Threat minus Safe for Anxious versus zero: $t(43)=3.504, \mathrm{p}=0.001,95 \% \mathrm{Cl}=0.0210 .078$, one-sample two-tailed $t$-test; Threat minus Safe for Controls versus zero: $t(87)=0.855, p=0.395,95 \% \mathrm{Cl}=-0.0280 .011$, onesample two-tailed $t$-test]. No other main effects or interactions were significant for any of the four feedback types (see Table 1 for full ANOVA tables). Our results rectify the original hypothesis by showing significantly increased punishment avoidance for anxious individuals (versus controls) during stress, but not safety.

\section{Modeling behavior}

Behavioral modeling may provide insights into the specific mechanism affected by stress in anxious individuals. To this end, we designed and confronted four models, each allowing different parameters related to punishment processing to be modulated by stress: learning rates, decision weights, feedback sensitivities, and feedback trends (see formal descriptions in the Supplementary Information). The four models, and a control model presuming no impact of stress on punishment processing, were fitted to behavior using a hierarchical Bayesian inference method ${ }^{8}$ (see Supplementary Information for details). The model including separate decision weights for punishment feedback trends was determined as the most likely model to explain the observed behaviors (Fig.1F). To validate this result, we first reproduced the behavioral effect by using the individually fitted model parameters to calculate selection probabilities in each trial [Fig. $1 \mathrm{H}$; Threat minus Safe for Anxious versus Controls: $t(130)=3.246, p=0.002,95 \% \mathrm{Cl}=0.010$ 0.040 , two-sample two-tailed $t$-test; Threat minus Safe for Anxious versus zero: $t(43)=3.864$, 
$\mathrm{p}<0.001,95 \% \mathrm{Cl}=0.0110 .035$, one-sample two-tailed $t$-test; Threat minus Safe for Controls versus zero: $t(87)=0.419, \mathrm{p}=0.676,95 \% \mathrm{Cl}=-0.0110 .007$, one-sample two-tailed $t$-test]. As a second validation, the correlation between the observed and the model-fitted behavioral effect was positive and significant [Fig.11; Pearson's $r=0.563, p<0.001$; Spearman's $\rho=0.601, p<0.001$; both two-tailed tests]. The model presumes that the behavioral effect is driven by larger punishment feedback trends in the Threat versus the Safe condition, and specifically so for participants in the Anxious group. Indeed, Anxious participants showed a higher punishment feedback trend in the Threat condition [Fig.1J; Anxious versus Controls: $t(130)=2.290, \mathrm{p}=0.024,95 \% \mathrm{Cl}=0.0670 .921$, two-sample two-tailed $t$-test], but not in the Safe condition [Fig.1K; Anxious versus Controls: $t(130)=0.377, p=0.707,95 \% \mathrm{Cl}=-0.3150 .463$, two-sample two-tailed $t$-test], and the differential punishment feedback trends (i.e. Threat minus Safe condition) also differed between the groups [Fig.1L; Anxious versus Controls: $t(130)=2.733, p=0.007,95 \% \mathrm{Cl}=0.1160 .725$, two-sample twotailed $t$-test]. Finally, the observed behavioral effect was positively and significantly correlated with the difference in punishment feedback trends between the Threat and the Safe condition [Fig.1M; Pearson's r=0.379, $p<0.001$; Spearman's $\rho=0.594, p<0.001$; both two-tailed tests]. We note that model used here (the punishment feedback trend model) also provides a better fit to the behavior when compared to the best models of the original study (Supplementary Fig.1). Therefore, these results show that the behavioral effect is reproduced by the model we employ here (Fig. $1 \mathrm{H}, \mathrm{I})$, and that it is directly related to the parameters designed to explain it (Fig.1M).

Overalal, the punishment feedback trend refers to the difference between the current and the previous feedback obtained for the selected option. As such, a punishment received following a sequence of (at least one) neutral outcomes, may contribute to subsequent avoidance decisions by decreasing the subjective utility of the selected option. Notably, because this model provided a better fit to behavior as compared to a model incorporating different learning rates, our results indicate that the observed avoidance bias is more likely due to an immediate short-lasting decision bias, rather than a slower and longer-lasting learning bias. As such, our results suggest that stress contributes to more impulsive avoidance behaviors in anxious individuals.

\section{Discussion}

Re-analyzing the data reported in Aylward et al. (2019) using behavioral models that are designed to address punishment-related biases, we observed that anxious participants were more likely to change their decisions following punishment-only feedback in a stressful condition. This result rectifies the hypothesis of the original study. While both studies analyzed the frequency of repeated selections, the original study combined reward-only and punishment-only feedbacks into the same analyses (i.e. frequentist and Bayesian ANOVAs), whereas we analyzed each feedback type separately.

Behavioral modeling revealed that the behavioral effect was related to anxious participants considering recent punishment feedback trends to a larger extent during stress. This effect could be considered an impulsive response to punishment during stress, rather than an aversive learning bias, because the latter depends on the slow integration of punishments across trials. While there could be an adaptive advantage in responding quickly to any aversive outcome in a 
potentially threatening and stressful context, such avoidance biases could lead to maladaptive behaviors when aversive outcomes occur independently of the context (as in the present study), or when information needs to be integrated across multiple experiences.

Notably, the ability to extrapolate recent feedback trends may depend on the integrity of prefrontal brain regions ${ }^{9,10}$. Because anxiety is associated with alterations in prefrontal cortex (PFC) function ${ }^{11-13}$, it could be fruitful to address the interactions between stress, anxiety, and punishment feedback trends with regards to PFC activity, as well as other PFC-dependent executive functions known to be affected by anxiety and stress, such as working memory and cognitive control ${ }^{14-18}$.

\section{Author contribution}

KCA and RP analyzed the data and wrote the manuscript.

\section{Competing interests}

The authors declare no competing interests. 
Figures
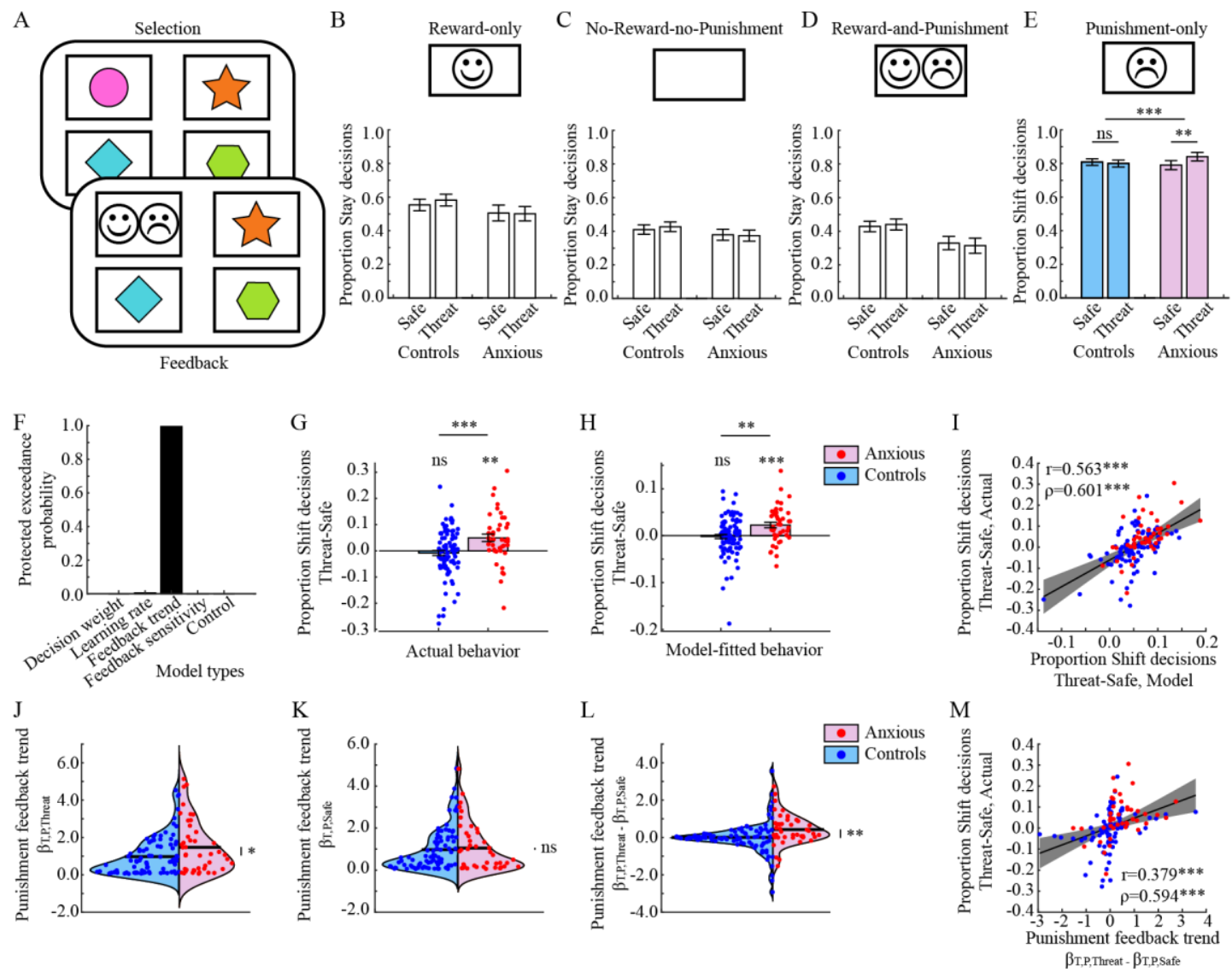

Figure 1. A. Schematic of the task. In each trial, participants selected one out of four presented bandits, after which probabilistic feedbacks consisting of smiley faces were presented on the selected bandits spatial location. Four different feedbacks were possible: a happy smiley face (reward-only, see B), no smiley face (no-reward-nopunishment, see C), a happy and a sad smiley face (reward-and-punishment, see $D$ ), and a sad smiley face (punishment-only, see E). B-E. The proportion of repeated selections following reward-only (B), no-reward-nopunishment (C), reward-and-punishment (D), and punishment-only (E), feedbacks. To conform with the common notions of Win-Stay and Lose-Shift responding, please observe that for B-D the proportion of repeated selections are displayed (i.e. in the case of reward-only feedback, this is equal to Win-Stay responding). By contrast, the proportion of Shift decisions (i.e. 1-proportion of Stay decisions) are shown for punishment-only feedback (i.e. this corresponds to Lose-Shift responding). F. The protected exceedance probability of a Bayesian model selection procedure indicates the probability that each model is the most likely model across all subjects taking into account the null possibility that differences in model evidence are due to chance. Accordingly, the model with separate parameters for feedback trends provided the most parsimonious fit to behavior. G-H. The interaction in E, displayed as the difference between the proportion of Shift decisions in the Threat versus the Safe condition for the actual behavior (G), and for the model-fitted behavior $(\mathbf{H})$. I. The correlation between the actually observed interaction (G) and the model-fitted interaction $(\mathrm{H})$. The correlation is highly significant, both when calculating the linear trend via Pearson's $r$ and the monotonically increasing trend via Spearman's $\rho$. Individuals in the Anxious group displayed a stronger punishment feedback trend in the Threat condition (J), while there was no group-difference in the Safe condition (K). The difference in punishment feedback trends between the Threat versus the Safe condition was larger for the Anxious group. M. Significant correlations between the actual interaction effect (see $\mathrm{G}$ ) and the difference in punishment 
feedback trends (see $L$ ), indicates that the observed effect relates to an individual's tendency to rely on punishment feedback trends during decision making. ${ }^{* *} p<0.001,{ }^{* *} p<0.01,{ }^{*} p<0.05$, ns not significant. r: Pearson's correlation coefficient, $\rho$ : Spearman's rank correlation coefficient.

Table 1. Repeated measures ANOVA for the analysis of repeated selections for the four different feedback types, with factors Group (Anxious, Control) and Condition (Threat, Safe).

\begin{tabular}{|c|c|c|c|c|c|c|}
\hline & $\begin{array}{l}\text { Sum of } \\
\text { Squares }\end{array}$ & $d f$ & $\begin{array}{l}\text { Mean } \\
\text { Square }\end{array}$ & $\mathrm{F}$ & $\begin{array}{c}\text { Uncorrected } \\
p \text {-value }\end{array}$ & $\eta_{p}^{2}$ \\
\hline \multicolumn{7}{|l|}{ Reward-only } \\
\hline Intercept & 67.297 & 1 & 67.297 & 347.11 & $<0.001$ & \\
\hline Group & 0.246 & 1 & 0.246 & 1.268 & 0.262 & 0.010 \\
\hline Error & 25.204 & 130 & 0.194 & & & \\
\hline Condition & 0.009 & 1 & 0.009 & 1.155 & 0.285 & 0.009 \\
\hline Group $\times$ Condition & 0.017 & 1 & 0.017 & 2.056 & 0.154 & 0.016 \\
\hline Error & 1.045 & 130 & 0.008 & & & \\
\hline \multicolumn{7}{|c|}{ No-reward-no-feedback } \\
\hline Intercept & 37.063 & 1 & 37.063 & 298.85 & $<0.001$ & \\
\hline Group & 0.103 & 1 & 0.103 & 0.830 & 0.364 & 0.006 \\
\hline Error & 16.122 & 130 & 0.124 & & & \\
\hline Condition & 0.002 & 1 & 0.002 & 0.444 & 0.506 & 0.003 \\
\hline Group $\times$ Condition & 0.007 & 1 & 0.007 & 1.984 & 0.161 & 0.015 \\
\hline Error & 0.455 & 130 & 0.004 & & & \\
\hline \multicolumn{7}{|c|}{ Reward-and-Punishment } \\
\hline Intercept & 33.578 & 1 & 33.578 & 218.16 & $<0.001$ & \\
\hline Group & 0.743 & 1 & 0.743 & 4.829 & 0.030 & 0.036 \\
\hline Error & 20.01 & 130 & 0.154 & & & \\
\hline Condition & $<0.001$ & 1 & $<0.001$ & 0.012 & 0.914 & $<0.001$ \\
\hline Group $\times$ Condition & 0.011 & 1 & 0.011 & 0.590 & 0.444 & 0.005 \\
\hline Error & 2.373 & 130 & 0.018 & & & \\
\hline \multicolumn{7}{|l|}{ Punishment-only } \\
\hline Intercept & 153.82 & 1 & 153.82 & 2433.9 & $<0.001$ & \\
\hline Group & 0.007 & 1 & 0.007 & 0.113 & 0.737 & $<0.001$ \\
\hline Error & 8.216 & 130 & 0.063 & & & \\
\hline
\end{tabular}




\begin{tabular}{lrrrrrr} 
Condition & 0.025 & 1 & 0.025 & 5.879 & 0.017 & 0.043 \\
Group x Condition & $\mathbf{0 . 0 4 9}$ & $\mathbf{1}$ & $\mathbf{0 . 0 4 9}$ & $\mathbf{1 1 . 5 7 7}$ & $<\mathbf{0 . 0 0 1} *$ & $\mathbf{0 . 0 8 2}$ \\
Error & 0.552 & 130 & 0.004 & & & \\
\hline
\end{tabular}

df: Degrees of Freedom. F: F-statistic. $\eta_{p}^{2}$ : Partial eta-squared. *: Significant after applying Bonferroni-correction ( $\alpha=0.0125$ ). 


\section{References}

1. Aylward, J., et al. Altered learning under uncertainty in unmedicated mood and anxiety disorders. Nature human behaviour 3, 1116-1123 (2019).

2. Duval, E.R., Javanbakht, A. \& Liberzon, I. Neural circuits in anxiety and stress disorders: a focused review. Therapeutics and clinical risk management 11, 115-126 (2015).

3. Herbert, J. Fortnighly review. Stress, the brain, and mental illness. Bmj 315, 530-535 (1997).

4. Hammen, C. Stress and depression. Annual review of clinical psychology 1, 293-319 (2005).

5. The Lancet Infectious, D. The intersection of COVID-19 and mental health. The Lancet. Infectious diseases 20, 1217 (2020).

6. Salari, N., et al. Prevalence of stress, anxiety, depression among the general population during the COVID-19 pandemic: a systematic review and meta-analysis. Globalization Health 16 (2020).

7. Rajkumar, R.P. COVID-19 and mental health: A review of the existing literature. Asian journal of psychiatry 52, 102066 (2020).

8. Piray, P., Dezfouli, A., Heskes, T., Frank, M.J. \& Daw, N.D. Hierarchical Bayesian inference for concurrent model fitting and comparison for group studies. PLoS computational biology 15, e1007043 (2019).

9. Beharelle, A.R., Polania, R., Hare, T.A. \& Ruff, C.C. Transcranial Stimulation over Frontopolar Cortex Elucidates the Choice Attributes and Neural Mechanisms Used to Resolve Exploration-Exploitation Trade-Offs. J Neurosci 35, 14544-14556 (2015).

10. Kovach, C.K., et al. Anterior Prefrontal Cortex Contributes to Action Selection through Tracking of Recent Reward Trends. J Neurosci 32, 8434-8442 (2012).

11. Davidson, R.J. Anxiety and affective style: role of prefrontal cortex and amygdala. Biological psychiatry 51, 68-80 (2002).

12. Park, J. \& Moghaddam, B. Impact of anxiety on prefrontal cortex encoding of cognitive flexibility. Neuroscience 345, 193-202 (2017).

13. Bishop, S.J. Neurocognitive mechanisms of anxiety: an integrative account. Trends in cognitive sciences 11, 307-316 (2007).

14. Moran, T.P. Anxiety and Working Memory Capacity: A Meta-Analysis and Narrative Review. Psychol Bull 142, 831-864 (2016).

15. Sandi, C. Stress and cognition. Wires Cogn Sci 4, 245-261 (2013).

16. Klein, K. \& Boals, A. The relationship of life event stress and working memory capacity. Appl Cognitive Psych 15, 565-579 (2001).

17. Liston, C., McEwen, B.S. \& Casey, B.J. Psychosocial stress reversibly disrupts prefrontal processing and attentional control. P Natl Acad Sci USA 106, 912-917 (2009).

18. Shields, G.S., Sazma, M.A. \& Yonelinas, A.P. The effects of acute stress on core executive functions: A meta-analysis and comparison with cortisol. Neurosci Biobehav R 68, 651-668 (2016). 